Short Note

\title{
Development of Simple Sequence Repeats (SSR) Markers in Setaria italica (Poaceae) and Cross-Amplification in Related Species
}

\author{
Heng-Sheng Lin, Chih-Yun Chiang, Song-Bin Chang * and Chang-Sheng Kuoh \\ Department of Life Sciences, Institute of Biodiversity, National Cheng Kung University, \\ Tainan 70101, Taiwan; E-Mails: t58601109520@gmail.com (H.-S.L); \\ chyuchiang@gmail.com (C.-Y.C); kuohpopo@gmail.com (C.-S.K.) \\ * Author to whom correspondence should be addressed; E-Mail: sbchang@ mail.ncku.edu.tw; \\ Tel.: +886-06-2757575-65529; Fax: +886-06-2742583.
}

Received: 14 October 2011; in revised form: 28 October 2011 / Accepted: 4 November 2011 / Published: 11 November 2011

\begin{abstract}
Foxtail millet is one of the world's oldest cultivated crops. It has been adopted as a model organism for providing a deeper understanding of plant biology. In this study, 45 simple sequence repeats (SSR) markers of Setaria italica were developed. These markers showing polymorphism were screened in 223 samples from 12 foxtail millet populations around Taiwan. The most common dinucleotide and trinucleotide repeat motifs are AC/TG $(84.21 \%)$ and CAT $(46.15 \%)$. The average number of alleles $\left(N_{\mathrm{a}}\right)$, the average heterozygosities observed $\left(H_{\mathrm{o}}\right)$ and expected $\left(H_{\mathrm{e}}\right)$ are $3.73,0.714,0.587$, respectively. In addition, 24 SSR markers had shown transferability to six related Poaceae species. These new markers provide tools for examining genetic relatedness among foxtail millet populations and other related species. It is suitable for germplasm management and protection in Poaceae.
\end{abstract}

Keywords: model system; Setaria italica; SSRs; Taiwan; transferability

\section{Introduction}

Foxtail millet (Setaria italica (L.) P. Beauv) is an old cereal consumed by people in Euraisa, Americas, Africa and Australia. It has also been an important crop for the indigenous tribes of Taiwan 
for thousands of years. It is able to endure droughts and grows quickly; therefore, the indigenous people cultivate foxtail millet instead of rice. Though several researches have used a few millet strains from Taiwan, little is known about the genetic diversity in hundreds of local landraces scattered throughout Taiwan [1,2].

With a relatively small genome $(515 \mathrm{Mb})$, the diploid foxtail millet has been adopted as model organism for providing deep understanding of the plant biology [3]. The importance of this species is rising since the project of its genome draft has been conducted by the Joint Genome Institute (JGI) of the US Department of Energy [4]. Compared to other familiar model systems, such as Arabidopsis (Arabidopsis thaliana), rice, or maize, large amounts of the genetic information were provided as an important resource for the research community. However, insufficient genetic resources of Setaria italica distributed around the world have been studied.

Several molecular markers in foxtail millets have been utilized including RFLP [1,3], RAPD [5], AFLP [6], and simple sequence repeats (SSR) [7,8]. These researches mainly focused on the species origin and the genetic map construction; no studies were conducted to assess the genetic diversity of the varying landraces in Taiwan.

Microsatellite, which is also called simple sequence repeats (SSR), are tandem repeat sequences of 1-6 base pairs of DNA. It has been proven to be useful in genetic diversity studies because of its high polymorphism, high variation, with abundant information and convenience; thus it is widely employed in many species [9-14]. Broadening the genetic base is regarded as a major task in species where inbreeding works have resulted in the decline of genetic diversity [15]. Several SSR markers have been developed by Jia et al. [7-8], as no one can ensure if novel SSR markers provide benefits or not. Reports have demonstrated that tetranucleotides are typically easier to be genotyped than di- and tri-nucleotide repeat SSRs [16]. To distinctly quantify the genetic variation among varying foxtail millets landraces in Taiwan, more SSR loci need to be developed.

In the present study, we report the identification of different types of SSR loci within representative landraces of foxtail millet by examining their polymorphism and cross-amplification in a further six related Poaceae species.

\section{Results and Discussion}

\subsection{Microsatellite Loci Isolation}

A total number of 570 genomic sequences from the RAPD-enriched library and 158 genomic sequences downloaded from the GenBank were screened for SSRs. In these sequences, 134 SSR among the 570 sequences and 16 SSR from the 158 GenBank sequences were found. A total of 150 SSR primer pairs were designed successfully by PRIMER3. Forty-five out of 150 SSR markers showed polymorphism among seven millets strains (NCKU.S.I.P1001, NCKU.S.I.P2001, NCKU.S.I.P3001, NCKU.S.I.P4001, NCKU.S.I.P5001, NCKU.S.I.P6001, NCKU.S.I.P7001) (Table 1). 
Table 1. Polymorphic simple sequence repeats (SSR) primers for Setaria italica.

\begin{tabular}{|c|c|c|c|c|c|c|c|c|}
\hline Locus & Repeat Sequence & & Primer Sequence $\left(5^{\prime}-3^{\prime}\right)$ & Ta $\left({ }^{\circ} \mathbf{C}\right)$ & Size & Accession No. & OS & PH \\
\hline \multirow[t]{2}{*}{ SITM02 } & $(\mathrm{TG})_{12}$ & $\mathrm{~F}$ & TAGTCGCTGGAAAGTTTCGG & 51.8 & 208 & JN565177 & - & No hit \\
\hline & & $\mathrm{R}$ & TAGTCGCTGGAAAGTTTCGG & & & & & \\
\hline \multirow[t]{2}{*}{ SITM04 } & $(\mathrm{TG})_{13}$ & $\mathrm{~F}$ & CGTGTCCTTGTACTCAGCCA & 53.8 & 240 & JN565179 & - & No hit \\
\hline & & $\mathrm{R}$ & CAATGGTCTCAGGTGTGGTG & & & & & \\
\hline \multirow[t]{2}{*}{ SITM05 } & $(\mathrm{GT})_{10}$ & $\mathrm{~F}$ & AGCTTACCCCTCACATTTAT & 47.7 & 204 & JN565180 & - & No hit \\
\hline & & $\mathrm{R}$ & ATGAGAAGGTGCCAAAATGC & & & & & \\
\hline \multirow{2}{*}{ SITM06 } & $(\mathrm{CA})_{10}$ & $\mathrm{~F}$ & GCTCTCTCCATCCCACATTC & 53.8 & 146 & JN565181 & - & No hit \\
\hline & & $\mathrm{R}$ & ТтСТССТТСССТТССТТТСС & & & & & \\
\hline \multirow[t]{2}{*}{ SITM07 } & $(\mathrm{AC})_{7 .} \cdot(\mathrm{CA})_{8}$ & $\mathrm{~F}$ & GCCCAAAAАCTCATTCTCCA & 49.7 & 55 & JN565182 & - & No hit \\
\hline & & $\mathrm{R}$ & ATAАСССТСАССАСТАСАAG & & & & & \\
\hline \multirow[t]{2}{*}{ SITM09 } & $(\mathrm{AC})_{15}$ & $\mathrm{~F}$ & CCCCTATGTTCCTTGGACCT & 53.8 & 207 & JN565184 & - & No hit \\
\hline & & $\mathrm{R}$ & GGAAAGCCAGTGTGAGTGCA & & & & & \\
\hline \multirow[t]{2}{*}{ SITM10 } & $(\mathrm{TCA})_{5}$ & $\mathrm{~F}$ & GGCTGGAGTGAGTCTTCGTC & 55.9 & 178 & JN565185 & - & No hit \\
\hline & & $\mathrm{R}$ & GCTGAGGAAAATGGTGAGGA & & & & & \\
\hline \multirow[t]{2}{*}{ SITM11 } & $(\mathrm{ATC})_{6}$ & $\mathrm{~F}$ & CTCGCCCATCTCTTCTTCAG & 53.8 & 113 & JN565186 & - & No hit \\
\hline & & $\mathrm{R}$ & CAAGCACAGGGAAGAGGAGT & & & & & \\
\hline \multirow[t]{2}{*}{ SITM14 } & $(\mathrm{CAT})_{15}$ & $\mathrm{~F}$ & TCTGAGGAGGAGGATGTGCT & 53.8 & 196 & JN565189 & - & No hit \\
\hline & & $\mathrm{R}$ & CATCTGAAGCAAACCTGAAT & & & & & \\
\hline \multirow[t]{2}{*}{ SITM15 } & $(\mathrm{ATC})_{8}$ & $\mathrm{~F}$ & TGGAACCGAAGCTGCCTACC & 55.9 & 223 & JN565190 & - & Sorghum bicolor \\
\hline & & $\mathrm{R}$ & AAGTCCAAGAAGTCGCCAGA & & & & & hypothetical protein \\
\hline \multirow[t]{2}{*}{ SITM17 } & $(\mathrm{AG})_{10}$ & $\mathrm{~F}$ & GCATACGGCTACTGGACATA & 51.8 & 109 & JN565192 & - & No hit \\
\hline & & $\mathrm{R}$ & ATCTTCTTTTGTTAGCGAGC & & & & & \\
\hline \multirow[t]{2}{*}{ SITM18 } & $(\mathrm{CAT})_{8}$ & $\mathrm{~F}$ & GCTCGCTAACAAAAGAAGAT & 47.7 & 72 & JN565193 & - & No hit \\
\hline & & $\mathrm{R}$ & AGGTTGAAATGAAGAAGAGG & & & & & \\
\hline \multirow[t]{2}{*}{ SITM19 } & $(\mathrm{TCA})_{8}$ & $\mathrm{~F}$ & CTTCCGCCATCAACCATTCG & 53.8 & 63 & JN565194 & - & No hit \\
\hline & & $\mathrm{R}$ & GACGAAGATGATGACGACGA & & & & & \\
\hline \multirow[t]{2}{*}{ SITM20 } & $(\mathrm{TGA})_{6}$ & $\mathrm{~F}$ & TGATGATGCCAATGAACCAG & 49.7 & 246 & JN565195 & - & No hit \\
\hline & & $\mathrm{R}$ & GCTATTTCCTACGCCCTTCC & & & & & \\
\hline \multirow[t]{2}{*}{ SITM22 } & $(\mathrm{ATG})_{7}$ & $\mathrm{~F}$ & TCCAAGTAGTGAAAGTGATA & 45.6 & 188 & JN565197 & - & No hit \\
\hline & & $\mathrm{R}$ & TTCCTCCTCGTCCTCTTCAT & & & & & \\
\hline \multirow[t]{2}{*}{ SITM23 } & $(\mathrm{ATG})_{5}$ & $\mathrm{~F}$ & ATGAAGAGGACGAGGAGGAA & 51.8 & 110 & JN565198 & - & No hit \\
\hline & & $\mathrm{R}$ & CGTTCCAGTAATATGTGCCC & & & & & \\
\hline \multirow[t]{2}{*}{ SITM24 } & $(\mathrm{ATG})_{7}$ & $\mathrm{~F}$ & AGGTCTGCTTGGGATGAAAT & 47.7 & 110 & JN565199 & - & No hit \\
\hline & & $\mathrm{R}$ & AACATTACCCCCTGAAGAAC & & & & & \\
\hline \multirow[t]{2}{*}{ SITM25 } & $(\mathrm{ATC})_{6}$ & $\mathrm{~F}$ & CTCGCCCATCTCTTCTTCAG & 53.8 & 113 & JN565200 & - & No hit \\
\hline & & $\mathrm{R}$ & CAAGCACAGGGAAGAGGAGT & & & & & \\
\hline \multirow[t]{2}{*}{ SITM26 } & $(\mathrm{TGA})_{15}$ & $\mathrm{~F}$ & TGAAGCAAACCTGAATCGTG & 49.7 & 186 & JN565201 & - & No hit \\
\hline & & $\mathrm{R}$ & TCTGAGGAGGAGGATGTGCT & & & & & \\
\hline \multirow[t]{2}{*}{ SITM27 } & $(\mathrm{CAT})_{20}$ & $\mathrm{~F}$ & TTTACAGCCAAGGAAGACGT & 49.7 & 221 & JN565202 & - & No hit \\
\hline & & $\mathrm{R}$ & GCTCCTCGATGGTATGCTCT & & & & & \\
\hline \multirow[t]{2}{*}{ SITM28 } & $(\mathrm{TGA})_{5}$ & $\mathrm{~F}$ & TAAGATGAGCGTTGGGGAGA & 51.8 & 101 & JN565203 & - & No hit \\
\hline & & $\mathrm{R}$ & ACGAACCGCACCAAATCTAC & & & & & \\
\hline \multirow[t]{2}{*}{ SITM30 } & $(\mathrm{ATG})_{7 \cdot(\mathrm{ATG})_{6}}$ & $\mathrm{~F}$ & TGTCGGAGATGATGAGGTGA & 51.8 & 220 & JN565205 & - & No hit \\
\hline & & $\mathrm{R}$ & GACGAACCGCATCAAATCTAA & & & & & \\
\hline
\end{tabular}


Table 1. Cont.

\begin{tabular}{|c|c|c|c|c|c|c|c|c|}
\hline Locus & Repeat Sequence & & Primer Sequence $\left(5^{\prime}-3^{\prime}\right)$ & $T_{\mathrm{a}}\left({ }^{\circ} \mathrm{C}\right)$ & Size & Accession No. & OS & PH \\
\hline \multirow[t]{2}{*}{ SITM32 } & $(\mathrm{GAT})_{6}$ & $\mathrm{~F}$ & CAGGATGACCAGGGAGATGC & 55.9 & 157 & JN565207 & - & No hit \\
\hline & & $\mathrm{R}$ & ACAGCTTTCCGCCTCAACCT & & & & & \\
\hline \multirow[t]{2}{*}{ SITM33 } & $(\mathrm{ATC})_{9}$ & $\mathrm{~F}$ & TTTGGACGACAGACGATTCA & 49.7 & 160 & JN565208 & - & No hit \\
\hline & & $\mathrm{R}$ & AAGTCCAAGAAGTCGCCAGA & & & & & \\
\hline \multirow[t]{2}{*}{ SITM34 } & $(\mathrm{CAT})_{5}$ & $\mathrm{~F}$ & AAGGGGTGGATGAGGTAGGT & 53.8 & 147 & JN565209 & - & Sorghum bicolor \\
\hline & & $\mathrm{R}$ & TCGAATTGAAGAAGAGCCTG & & & & & hypothetical protein \\
\hline \multirow[t]{2}{*}{ SITM37 } & $(\mathrm{GAT})_{7}$ & $\mathrm{~F}$ & CATCGTTGTAAGAAGTGGAA & 47.7 & 166 & JN565212 & - & No hit \\
\hline & & $\mathrm{R}$ & CTTTTTGGCTGCTGGGTTT & & & & & \\
\hline \multirow[t]{2}{*}{ SITM38 } & $(\mathrm{TCA})_{9}$ & $\mathrm{~F}$ & ACGGAAGAGGCAGTCACAAT & 51.8 & 206 & JN565213 & - & No hit \\
\hline & & $\mathrm{R}$ & ATTGGTGATGGATTCGTCAT & & & & & \\
\hline \multirow[t]{2}{*}{ SITM40 } & $(\mathrm{ATC})_{9}$ & $\mathrm{~F}$ & GTTGCTGCTGATGCTTGGT & 51.1 & 219 & JN565215 & - & No hit \\
\hline & & $\mathrm{R}$ & AATGCGAATCTCTTGGTGCT & & & & & \\
\hline \multirow[t]{2}{*}{ SITM41 } & $(\mathrm{ATC})_{5}$ & $\mathrm{~F}$ & GGTTTCCTTCCCCTTGTGTT & 51.8 & 87 & JN565216 & - & No hit \\
\hline & & $\mathrm{R}$ & CGGTCCCTATTGTTGATGAT & & & & & \\
\hline \multirow[t]{2}{*}{ SITM42 } & $(\mathrm{ATG})_{8}$ & $\mathrm{~F}$ & TGTTCATGCGGATTTTCTTG & 47.7 & 169 & JN565217 & - & No hit \\
\hline & & $\mathrm{R}$ & GGGACTCGGCAAAATAATCA & & & & & \\
\hline \multirow[t]{2}{*}{ SITM44 } & $(\text { TTA })_{5}$ & $\mathrm{~F}$ & TCGGTTAATGCCTTTTGCTC & 49.7 & 70 & JN565219 & - & No hit \\
\hline & & $\mathrm{R}$ & TTATGGACGGAAATGGTGTG & & & & & \\
\hline \multirow[t]{2}{*}{ SITM46 } & $(\mathrm{TGA})_{6}$ & $\mathrm{~F}$ & TGCCGAAAGGATCAAAAAGA & 47.7 & 215 & JN565221 & - & No hit \\
\hline & & $\mathrm{R}$ & TCACCACTGCCATCATCACT & & & & & \\
\hline \multirow[t]{2}{*}{ SITM49 } & $(\mathrm{TG})_{10} \cdot(\mathrm{GT})_{18}$ & $\mathrm{~F}$ & AGGTATCGTGCGTGTGTCTG & 53.8 & 82 & JN565224 & - & No hit \\
\hline & & $\mathrm{R}$ & AATGATGAATAATGGTGCTG & & & & & \\
\hline \multirow[t]{2}{*}{ SITM51 } & $(\mathrm{AC})_{13}$ & $\mathrm{~F}$ & CAATGGTCTCAGGTGTGGTG & 53.8 & 164 & JN565226 & - & No hit \\
\hline & & $\mathrm{R}$ & TACCATTCATCAAAAGTGCC & & & & & \\
\hline \multirow[t]{2}{*}{ SITM53 } & $(\mathrm{GT})_{9 \cdot}(\mathrm{GT})_{10}$ & $\mathrm{~F}$ & GTCACTTGTTGTTGTTGCGA & 49.7 & 158 & JN565228 & - & No hit \\
\hline & & $\mathrm{R}$ & GAACACGGAGAAGCGAAAAG & & & & & \\
\hline \multirow[t]{2}{*}{ SITM55 } & $(\mathrm{AC})_{14}$ & $\mathrm{~F}$ & GTCGTAGCTTTCGGTCCAAC & 53.8 & 196 & JN565230 & - & No hit \\
\hline & & $\mathrm{R}$ & CTGGGAATAGAAGAACATGC & & & & & \\
\hline \multirow[t]{2}{*}{ SITM57 } & $(\mathrm{AC})_{13}$ & $\mathrm{~F}$ & GGGTAGTGGTCTGGTGGTCA & 55.9 & 196 & JN565232 & - & No hit \\
\hline & & $\mathrm{R}$ & GTATCACTTCAGGCGGCATT & & & & & \\
\hline \multirow[t]{2}{*}{ SITM59 } & $(\mathrm{TG})_{22}$ & $\mathrm{~F}$ & AGGAAGGGGAAACACTGACC & 53.8 & 158 & JN565234 & - & Glycine max isolate \\
\hline & & $\mathrm{R}$ & GCGTTGTTGTTCATCGTGTT & & & & & $\begin{array}{c}\text { RG10 lipoxygenase } 2 \\
\text { (Lx2) }\end{array}$ \\
\hline \multirow[t]{2}{*}{ SITM62 } & $(\mathrm{AC})_{15 .} \cdot(\mathrm{AC})_{6}$ & $\mathrm{~F}$ & CGAACCGCTCACAAACACTA & 51.8 & 149 & JN565237 & - & No hit \\
\hline & & $\mathrm{R}$ & TAGTTGGAGAAGTTGAGTGC & & & & & \\
\hline \multirow[t]{2}{*}{ SITM65 } & $(\mathrm{GT})_{13}$ & $\mathrm{~F}$ & GCCACCCCTTGATTGTTATG & 51.8 & 228 & JN565240 & - & No hit \\
\hline & & $\mathrm{R}$ & GCTCAACATCTGGCATTTCA & & & & & \\
\hline \multirow[t]{2}{*}{ SITM68 } & $(\mathrm{GT})_{26}$ & $\mathrm{~F}$ & GGCATTGGACGAGTTACGGC & 55.9 & 108 & JN565243 & - & No hit \\
\hline & & $\mathrm{R}$ & GTCATAGCTCACGGCACAAC & & & & & \\
\hline \multirow[t]{2}{*}{ SITM73 } & $(\mathrm{CT})_{21}$ & $\mathrm{~F}$ & CCTGAACTGGTTGGAGTTGG & 53.8 & 243 & JN565248 & - & No hit \\
\hline & & $\mathrm{R}$ & ATCAGGACCAAGGGCAAAAT & & & & & \\
\hline
\end{tabular}


Table 1. Cont.

\begin{tabular}{|c|c|c|c|c|c|c|c|c|}
\hline Locus & Repeat Sequence & & Primer Sequence $\left(5^{\prime}-3^{\prime}\right)$ & $\boldsymbol{T}_{\mathrm{a}}\left({ }^{\circ} \mathbf{C}\right)$ & size & Accession No. & OS & PH \\
\hline \multirow[t]{2}{*}{ SITM84 } & $\begin{array}{l}(\mathrm{CT})_{3} \cdot(\mathrm{AT})_{3} \cdot(\mathrm{GA})_{4} \cdot \\
(\mathrm{GA})_{7} \cdot(\mathrm{GGC})_{3}\end{array}$ & $\mathrm{~F}$ & TCGGTCCTTCACCTTCTTTG & 51.8 & 110 & JN565259 & EF117799 & No hit \\
\hline & & $\mathrm{R}$ & CGCCATCACCTTCTCCTCGC & & & & & \\
\hline \multirow[t]{2}{*}{ SITM86 } & $\begin{array}{l}(\mathrm{AT})_{3} \cdot(\mathrm{CG})_{3} \cdot(\mathrm{CG})_{3} \cdot \\
(\mathrm{CGC})_{7} \cdot(\mathrm{CG})_{3}\end{array}$ & $\mathrm{~F}$ & CTTGCTTAGATCTGGACTAA & 47.7 & 202 & JN565261 & EF117797 & No hit \\
\hline & & $\mathrm{R}$ & GCGAGGCTGGAGATAGTCAG & & & & & \\
\hline \multirow[t]{2}{*}{ SITM91 } & $(\mathrm{GGC})_{4}$ & $\mathrm{~F}$ & GTTCGCAGCAGCACTCATTA & 51.8 & 161 & JN565266 & - & No hit \\
\hline & & $\mathrm{R}$ & TTGCATGTGCAGGTATAGGC & & & & & \\
\hline
\end{tabular}

F: forward primer; R: reverse primer; Ta: annealing temperature; Size: expected size of PCR products (bp); OS: original sequences retrieved from Genbank; PH: putative homology.

\subsection{Characterization of Microsatellite Loci}

Among these 45 SSRs, 19 contained dinucleotide repeats, and 26 contained trinucleotide . The most common dinucleotide repeats motif was AC/TG (84.21\%). CAT was the most common trinucleotide repeat motif in foxtail millet (46.15\%). However, according to research of EST-SSR markers of foxtail millet, the most common repeat motifs were TC/AG [7] and CAG/TCT [8]. The most common repeats in wheat are CA or TG, also GA [17] or GT repeats [18]. In other monocot crops such as barley, wheat, maize, sorghum and rice, the most common trinucleotide repeats were CCG/GGC or AAC/TTG [19]. The differences may be caused by different genomes being tested or our use of different SSR isolation strategies with varying affinities.

\subsection{Genetic Characterization of Microsatellite Loci}

Forty-five SSRs were PCR amplified in 223 samples from 12 collection sites to assess the diversity of foxtail millet in Taiwan. Characterizations of these loci are summarized in Table 2. The average number of alleles $\left(N_{\mathrm{a}}\right)$ ranged from 1 to 8 , with an average of 3.73. The average observed heterozygosities $\left(H_{\mathrm{o}}\right)$ ranged from 0 to 0.886 with an average of 0.714 . The expected heterozygosities $\left(H_{\mathrm{e}}\right)$ ranged from 0 to 0.813 , with an average of 0.587 . No linkage disequilibrium was observed from pairwise comparisons of loci. 32 SSR loci significantly deviated from Hardy-Weinberg equilibrium $\left(H_{\mathrm{W}}\right)$ (Table 2 and Table 1S), which were assumed to be a result of long time isolation of the foxtail millet population in Taiwan. GenBank (BLASTX) searches indicated that three SSR loci among the 45 SSR markers, including SITM 15, SITM34 and SITM59 have putative function at E values less than $10^{-05}$ (Table 1).

Table 2. Results of diversity estimation in 223 samples of Setaria italica in Taiwan.

\begin{tabular}{cccccccccc}
\hline Locus & $\boldsymbol{N}_{\mathbf{a}}$ & $\boldsymbol{H}_{\mathbf{0}}$ & $\boldsymbol{H}_{\mathbf{e}}$ & $\boldsymbol{H}_{\mathrm{W}}$ & Locus & $\boldsymbol{N}_{\mathbf{a}}$ & $\boldsymbol{H}_{\mathbf{0}}$ & $\boldsymbol{H}_{\mathbf{e}}$ & $\boldsymbol{H}_{\mathrm{W}}$ \\
\hline SITM02 & 5 & 0.701 & 0.75 & $* *$ & SITM33 & 3 & 0.76 & 0.625 & ND \\
SITM04 & 7 & 0.883 & 0.647 & $* * *$ & SITM34 & 7 & 0.781 & 0.653 & ND \\
SITM05 & 6 & 0.886 & 0.64 & $* * *$ & SITM37 & 1 & 0 & 0 & ND \\
SITM06 & 3 & 0.786 & 0.533 & $* * *$ & SITM38 & 2 & 0.828 & 0.371 & ND \\
SITM07 & 4 & 0.726 & 0.607 & $* * *$ & SITM40 & 2 & 0.753 & 0.653 & ND \\
SITM09 & 5 & 0.757 & 0.629 & $* * *$ & SITM41 & 1 & 0 & 0 & ND \\
SITM10 & 3 & 0.744 & 0.736 & $* * *$ & SITM42 & 4 & 0.773 & 0.52 & $* * *$ \\
\hline
\end{tabular}


Table 2. Cont.

\begin{tabular}{cccccccccc}
\hline Locus & $\boldsymbol{N}_{\mathbf{a}}$ & $\boldsymbol{H}_{\mathbf{0}}$ & $\boldsymbol{H}_{\mathbf{e}}$ & $\boldsymbol{H}_{\mathbf{W}}$ & Locus & $\boldsymbol{N}_{\mathbf{a}}$ & $\boldsymbol{H o}$ & $\boldsymbol{H}_{\mathbf{e}}$ & $\boldsymbol{H}_{\mathbf{W}}$ \\
\hline SITM11 & 3 & 0.719 & 0.75 & $* * *$ & SITM44 & 7 & 0.774 & 0.61 & $* * *$ \\
SITM14 & 6 & 0.783 & 0.639 & $* * *$ & SITM46 & 3 & 0.723 & 0.57 & $* * *$ \\
SITM15 & 4 & 0.788 & 0.62 & $* * *$ & SITM49 & 8 & 0.801 & 0.687 & $* * *$ \\
SITM17 & 3 & 0.792 & 0.75 & $* * *$ & SITM51 & 7 & 0.875 & 0.653 & $* * *$ \\
SITM18 & 2 & 0.704 & 0.813 & $* *$ & SITM53 & 4 & 0.673 & 0.625 & ND \\
SITM19 & 3 & 0.783 & 0.764 & ND & SITM55 & 6 & 0.707 & 0.653 & $* * *$ \\
SITM20 & 2 & 0.873 & 0.653 & $* * *$ & SITM57 & 6 & 0.683 & 0.575 & $* * *$ \\
SITM22 & 2 & 0.76 & 0.667 & $* *$ & SITM59 & 3 & 0.74 & 0.75 & $* * *$ \\
SITM23 & 2 & 0.726 & 0.625 & ND & SITM62 & 3 & 0.743 & 0.652 & $* * *$ \\
SITM24 & 3 & 0.782 & 0.778 & $* * *$ & SITM65 & 3 & 0.747 & 0.588 & $* * *$ \\
SITM25 & 3 & 0.702 & 0.694 & ND & SITM68 & 2 & 0.757 & 0.487 & $* * *$ \\
SITM26 & 1 & 0 & 0 & $* * *$ & SITM73 & 4 & 0.731 & 0.563 & $* * *$ \\
SITM27 & 2 & 0.838 & 0.569 & ND & SITM84 & 4 & 0.817 & 0.736 & $* * *$ \\
SITM28 & 7 & 0.75 & 0.468 & $* *$ & SITM86 & 2 & 0.69 & 0.478 & $* * *$ \\
SITM30 & 2 & 0.794 & 0.468 & $* *$ & SITM91 & 4 & 0.722 & 0.625 & ND \\
SITM32 & 4 & 0.783 & 0.542 & ND & & & & & \\
\hline \multicolumn{7}{c}{$* * 005 * * * p<0.01 ;$ ND non-significant deviation. } & &
\end{tabular}

\subsection{Cross-Amplification of SSR Loci in Related Poaceae Species}

Cross-species amplification with the 45 SSR primers obtained from Setaria italica were applied to six other related species $(N=18)$, including Hygroryza aristata (Retz.) Nees (Asian watergrass), Setaria plicata (Lamk.) T cooke (Small palm grass), Microstegium vimineum (Trin.) A camus (Flexible sasa grass), Oplimenus compositus (L.) P. Beauv (Armgrass), Cynodon dactylon (L.) Pers (Bermuda Grass), and Setaria verticillata (L.) P. Beauv (Hooked Bristlegrass). Finally, 24 primers could yield PCR products in the other Poaceae species, indicating transferability of the markers (53\%) (Table 3).

Table 3. Cross-amplification in six related Poaceae species.

\begin{tabular}{ccccccccccccccc}
\hline Locus & A & $\mathbf{B}$ & $\mathbf{C}$ & $\mathbf{E}$ & $\mathbf{F}$ & $\mathbf{G}$ & Locus & $\mathbf{A}$ & $\mathbf{B}$ & $\mathbf{C}$ & $\mathbf{E}$ & $\mathbf{F}$ & $\mathbf{G}$ \\
\hline SITM02 & - & - & - & - & - & - & SITM33 & - & - & - & - & - & - \\
SITM04 & + & + & + & + & + & + & SITM34 & - & - & - & - & - & - \\
SITM05 & + & + & + & + & + & + & SITM37 & - & - & - & - & - & - \\
SITM06 & + & + & + & + & + & + & SITM38 & + & + & + & + & + & + \\
SITM07 & + & + & + & + & + & + & SITM40 & - & - & - & - & - & - \\
SITM09 & + & + & + & + & + & + & SITM41 & + & + & + & + & + & + \\
SITM10 & + & + & + & + & + & + & SITM42 & - & - & - & - & - & - \\
SITM11 & + & + & + & + & + & + & SITM44 & - & - & - & - & - & - \\
SITM14 & - & - & - & - & - & - & SITM46 & - & - & - & - & - & - \\
SITM15 & - & - & - & - & - & - & SITM49 & + & + & + & + & + & + \\
SITM17 & + & + & + & + & + & + & SITM51 & - & - & - & - & - & - \\
SITM18 & + & + & + & + & + & + & SITM53 & - & - & - & - & - & - \\
SITM19 & + & + & + & + & + & + & SITM55 & - & - & - & - & - & - \\
SITM20 & + & + & + & + & + & + & SITM57 & - & - & - & - & - & - \\
SITM22 & + & + & + & + & + & + & SITM59 & - & - & - & - & - & - \\
\hline
\end{tabular}


Table 3. Cont.

\begin{tabular}{|c|c|c|c|c|c|c|c|c|c|c|c|c|c|}
\hline Locus & $\mathbf{A}$ & B & $\mathbf{C}$ & $\mathbf{E}$ & $\mathbf{F}$ & $\mathbf{G}$ & Locus & $\mathbf{A}$ & B & C & $\mathbf{E}$ & $\mathbf{F}$ & G \\
\hline SITM23 & + & + & + & + & + & + & SITM62 & + & + & + & + & + & + \\
\hline SITM24 & + & + & + & + & + & + & SITM65 & + & + & + & + & + & + \\
\hline SITM25 & + & + & + & + & + & + & SITM68 & - & - & - & - & - & - \\
\hline SITM26 & + & + & + & + & + & + & SITM73 & - & - & - & - & - & - \\
\hline SITM27 & + & + & + & + & + & + & SITM84 & - & - & - & - & - & - \\
\hline SITM28 & - & - & - & - & - & - & SITM86 & + & + & + & + & + & + \\
\hline SITM30 & - & - & - & - & - & - & SITM91 & + & + & + & + & + & + \\
\hline SITM32 & - & - & - & - & - & - & & & & & & & \\
\hline
\end{tabular}

+: successful amplification with expected allele size; -: absence of amplification; A: Hygroryza aristata (Retz.) Nees; B: Setaria plicata (Lamk.) T cooke; C: Microstegium vimineum (Trin.) A camus; E: Oplimenus compositus (L.) P. Beauv; F: Cynodon dactylon (L.)Pers.; G: Setaria verticillata (L.) P. Beauv.

\section{Experimental Section}

\subsection{Samples Collection}

A total of 223 samples of Setaria italica were collected from 12 sites in Taiwan. Six Poaceae species each with 3 samples were collected for cross-species amplification. All samples of the tested materials were listed in Table 4. Genomic DNA was isolated from leaf tissue of each individual using a DNeasy Plant Mini Kit (Qiagen, Hilden, Germany). 
Table 4. Information on voucher specimens for Setaria italica and the other related Poaceae species. GPS coordinates are provided. All samples in this research were collected by H.-S. Lin and deposited in the Institute of Biodiversity, Department of Life Science, National Cheng Kung University, Taiwan.

\begin{tabular}{|c|c|c|c|c|}
\hline Pop & Taxon & Collection Sites & GPS Coordinates $(\mathbf{N}, \mathbf{E})$ & Collection Number \\
\hline $\mathrm{P} 1$ & Setaria italica (L.) P. Beauv & Jianshi Township, Hsinchu County, Taiwan & $24.675722^{\circ}, 121.208725^{\circ}$ & NCKU.S.I.P1001 NCKU.S.I.P10020 \\
\hline P3 & Setaria italica (L.) P. Beauv & Ren'ai Township, Nantou County, Taiwan & $24.012599^{\circ}, 121.124954^{\circ}$ & NCKU.S.I.P3001 NCKU.S.I.P30018 \\
\hline P5 & Setaria italica (L.) P. Beauv & Xinyi Township, Nantou County, Taiwan & $23.621878^{\circ}, 120.882912^{\circ}$ & NCKU.S.I.P5001 NCKU.S.I.P50017 \\
\hline P6 & Setaria italica (L.) P. Beauv & Yanping Township, Taitung County, Taiwan & $22.894283^{\circ}, 121.062212^{\circ}$ & NCKU.S.I.P6001 NCKU.S.I.P60016 \\
\hline P7 & Setaria italica (L.) P. Beauv & Daren Township, Taitung County, Taiwan & $22.269876^{\circ}, 120.852871^{\circ}$ & NCKU.S.I.P7001 NCKU.S.I.P70020 \\
\hline & Setaria italica (L.) P. Beauv & ManzHou Township, Pingtung County, Taiwan & $22.109498^{\circ}, 120.873299^{\circ}$ & S.I.P100016 \\
\hline P1 & Setaria italica (L.) P. Beauv & Lanyu Township, Taitung County, Taiwan & $22.057005^{\circ}, 121.562519^{\circ}$ & NCKU.S.I.P11001 NCKU.S.I.P110023 \\
\hline & Setaria italica (L.) P. Beauv & Lanyu Township, Taitung County, Taiwan & $22.055812^{\circ}, 121.515269^{\circ}$ & NCKU.S.I.P12001 NCKU.S.I.P120018 \\
\hline & Hygroryza aristata (Retz.) Nees & Liuying Dist., Tainan City, Taiwan & $23.265053^{\circ}, 120.332919^{\circ}$ & NCKU.H.A.001 NCKU.H.A.003 \\
\hline & Setaria plicata (Lamk.) T cooke & Ren'ai Township, Nantou County, Taiwan & $22.272418^{\circ}, 120.843773^{\circ}$ & NCKU.S.P.001 NCKU.S.P.003 \\
\hline
\end{tabular}




\subsection{Development and Screening of SSR Markers}

The strategies in this study are based on PCR isolation of microsatellite arrays (PIMA), which began with an enriched pool of small DNA fragments amplified using RAPD primers [20]. PCR amplification was performed in $20 \mu \mathrm{L}$ volume containing $20 \mathrm{ng}$ of genomic DNA, $0.2 \mathrm{mM}$ of dNTPs, $2 \mathrm{mM} \mathrm{MgCl}_{2}, 0.2 \mathrm{U}$ Go-Taq polymerase (Promega, Madison, Wisconsin, USA), and 5 pmol of one RAPD primers. Five-hundred RAPD primers were used to construct randomly amplified fragments library (MDBIO, Piscataway, New Jersey, USA). Reactions were run on an MyCyclerTM Thermal Cycler (BIO-RAD, Benicia, California, USA) using the following conditions: 3 min of denaturation at $94{ }^{\circ} \mathrm{C}$, followed by 45 cycles at $94{ }^{\circ} \mathrm{C}$ for $1 \mathrm{~min}$, annealing temperature specific to each primer for $1 \mathrm{~min}$, extension at $72{ }^{\circ} \mathrm{C}$ for $2 \mathrm{~min}$, and a final extension at $72{ }^{\circ} \mathrm{C}$ for $5 \mathrm{~min}$. Amplification products were analyzed in the electropHoresis (2\% agarose gel using 100-bp ladder molecular size standard) (Geneaid, Taipei, Taiwan) to evaluate the allele size through ethidium bromide staining. The amplified DNA fragments with the size of 200-2000 bp were extracted using the Gel Extraction kit (Geneaid, Taipei, Taiwan). DNA fragments were ligated into a p-GEM-T Easy Vector following the manufacturer's instruction and the plasmids were transformed into Escherichia coli cells (Promega, Madison, Wisconsin, USA). Each clone was screened using repeat-specific primers including $(\mathrm{AC})_{5},(\mathrm{AG})_{5},(\mathrm{AT})_{5},(\mathrm{CG})_{5},(\mathrm{CT})_{5}$ and $(\mathrm{GT})_{5}$ and 2 vector primers including forward M13 (5'-dGTTTTCCCAGTCACGAC-3') and reverse M13 (5'-dGTTTTCCCAGTCACGAC-3') primers. The conditions of colony-PCR are $3 \mathrm{~min}$ at $94{ }^{\circ} \mathrm{C}$, followed by 45 cycles at $94{ }^{\circ} \mathrm{C}$ for $1 \mathrm{~min}$, annealing at $53{ }^{\circ} \mathrm{C}$ for $1 \mathrm{~min}, 2 \mathrm{~min}$ at $72{ }^{\circ} \mathrm{C}$, and $5 \mathrm{~min}$ at $72{ }^{\circ} \mathrm{C}$. In positive clones, a DNA fragment which contains a SSR appears as a band on the gel. Plasmid DNA of positive clones was purified using the Plasmid Miniprep Kit (BioKit, Miaoli, Taiwan). Ten $\mu \mathrm{L}$ of plasmid DNA with a concentration of $100 \mathrm{ng} / \mu \mathrm{L}$ was used in each sequencing reaction. DNA sequencing in both directions of the insert DNA was conducted using an Applied Biosystems 3730 DNA Analyzer with BigDyeR Terminator v3.1 Cycle Sequencing Kit (Applied Biosystems, Foster, California, USA). There were 134 SSRs among 570 positive clones examined.

To obtain additional useful SSR markers, 158 genomic DNA sequences of foxtail millets were downloaded from GenBank [21]. The criteria of no less than 16 repeat units for mono-, nine for di-, five for tri- to hexa-nucleotide repeats in perfect SSR and no less than $12 \mathrm{bp}$ for imperfect SSR were adopted. Finally, a total of 150 SSR primer pairs were designed using PRIMER3 software for detecting the diversity of foxtail millet in Taiwan [22].

\subsection{Characterization of Developed SSR Primers}

To evaluate the usefulness of SSRs, PCR reactions were performed on $20 \mu \mathrm{L}$ volumes containing $10 \mathrm{ng}$ of genomic DNA, $0.2 \mathrm{mM}$ dNTPs, $2 \mathrm{mM} \mathrm{MgCl}$, $0.2 \mathrm{U}$ Go-Taq polymerase and $0.12 \mu \mathrm{m}$ of each primer. PCR conditions were as follows: $3 \mathrm{~min}$ at $94{ }^{\circ} \mathrm{C}$, followed by 35 cycles at $94{ }^{\circ} \mathrm{C}$ for $1 \mathrm{~min}$, annealing temperature specific to each primer for $1 \mathrm{~min}, 1 \mathrm{~min}$ at $72{ }^{\circ} \mathrm{C}$, and $5 \mathrm{~min}$ at $72{ }^{\circ} \mathrm{C}$.

The average number of allele (Na), the average observed (Ho), and expected heterozygosities (He) were calculated using the software CERVUS 3.0 [23]. Test of deviation of Hardy-Weinberg equilibrium $\left(H_{\mathrm{W}}\right)$ and linkage disequilibrium $(\mathrm{LD})$ were performed using the GenePop program [24]. 
The sequences were searched against the GenBank nucleotide collection database using TBLASTX for functional annotation with a thresHold of E-value $<1.00 \mathrm{E}^{-05}$.

\subsection{Cross-Amplification of Developed SSR Markers}

Furthermore, cross-species amplification of the SSR primers obtained from Setaria italica were applied to six other related species $(N=18)$. SSR markers were PCR amplified on $20 \mu \mathrm{L}$ volumes containing $10 \mathrm{ng}$ of genomic DNA, $0.2 \mathrm{mM}$ dNTPs, $2 \mathrm{mM} \mathrm{MgCl} 2,0.2 \mathrm{U}$ Go-Taq polymerase and $0.12 \mu \mathrm{m}$ of each primer. The conditions are carried out as following: $3 \mathrm{~min}$ at $94{ }^{\circ} \mathrm{C}, 35$ cycles at $94{ }^{\circ} \mathrm{C}$ for $1 \mathrm{~min}$, annealing temperature specific to each primer for $1 \mathrm{~min}, 1 \mathrm{~min}$ at $72{ }^{\circ} \mathrm{C}$, and $5 \mathrm{~min}$ at $72{ }^{\circ} \mathrm{C}$.

\section{Conclusions}

In summary, these 45 novel SSR markers of foxtail millet showed polymorphism and transferability to the related Poaceae species in Taiwan. They can be used as molecular markers for application of population genetics, breeding and further landraces identification.

\section{References}

1. Fukunaga, K.; Wang, Z.; Kato, K.; Kawase, M. Geographical variation of nuclear genome RFLPs and genetic differentiation of foxtail millet, Setaria italica (L.) P. Beauv. Genet. Resour. Crop Evol. 2002, 49, 95-101.

2. Hirano, R.; Naito, K.; Fukunaga, K.; Watanabe, K.N.; Ohsawa, R.; Kawase, M. Genetic structure of landraces in foxtail millet (Setaria italica (L.) P. Beauv.) revealed with transposon display and interpretation to crop evolution of foxtail millet. Genome 2011, 54, 498-506.

3. Wang, Z.M.; Devos, K.M.; Liu, C.J.; Wang, R.Q.; Gale, M.D. Construction of RFLP-based maps of foxtail millet, Setaria italica (L.) P. Beauv. Theor. Appl. Genet. 1998, 96, 31-36.

4. Doust, A.N.; Elizabeth, A.K.; Katrien, M.D.; Jeffery, L.B. Foxtail millet: A sequence-driven grass model system. Plant Physiol. 2009, 149, 137-141.

5. ScHontz, D.; Rether, B. Genetic variability in foxtail millet, Setaria italica (L.) P. Beauv: Identification and classification of lines with RAPD markers. Plant Breed. 1999, 118, 190-192.

6. Le Thierry D'ennequin, M.; Panaud, O.; Toupance, B. Assessment of genetic relationships between Setaria italica and its wild relatives $S$. viridis using AFLP marker. Theor. Appl. Genet. 2000, 100, 1061-1066.

7. Jia, X.; Shi, Y.S.; Song, Y.C.; Wang, G.Y.; Wang, T.Y.; Li, Y. Development of EST-SSR in foxtail millet (Setaria italica). Genet. Resour. Crop Evol. 2007, 54, 233-236.

8. Jia, X.; Zhang, Z.; Liu, Y.; Zhang, C.; Shi, Y.; Song, Y.; Wang, T.; Li, Y. Development and genetic mapping of SSR markers in foxtail millet [Setaria italica (L.) P. Beauv.]. Theor. Appl. Genet. 2009, 118, 821-829.

9. Gupta, P.K.; Balyan, H.S.; Sharma, P.C.; Ramesh, B. Microsatellites in plants: A new class of molecular marker. Curr. Sci. 1996, 70, 45-54.

10. Jarne, P.; Lagoda, P.J.L. Microsatellites, form molecules to populations and back. Trends Ecol. Evol. 1996, 11, 424-429. 
11. Knapik, E.W.; Goodman, A.; Ekker, M.; Chevrette, M.; Delgado, J.; Neuhauss, S.; Shimoda, N.; Driever, W.; Fishman, M.C.; Jacob, H.J. A microsatellite genetic linkage map for zebrafish (Danio rerio). Nat. Genet. 1998, 18, 338-343.

12. Cipriani, G.; Lot, G.; Huang, W.G.; Marrazzo, M.T.; Peterlunger, E.; Testolin, R. AC/GT and AG/CT microsatellite repeats in peach [Prunus persica (L) Batsch]: Isolation, characterization and cross-species amplification in Prunus. Theor. Appl. Genet. 1999, 99, 65-72.

13. Metais, I.; Hamon, B.; Jalouzot, R.; Peltier, D. Structure and level of genetic diversity in various bean types evidenced with microsatellite markers isolated from a genomic enriched library. Theor. Appl. Genet. 2002, 104, 1346-1352.

14. Zane, L.; Bargelloni, L.; Patarnello, T. Strategies for microsatellite isolation: A review. Mol. Ecol. 2002, 11, 1-16.

15. Graham, J.; Mcnicol, R.J.; Mcnicol, J.W. A comparison of methods for the estimation of genetic diversity in strawberry cultivars. Theor. Appl. Genet. 1996, 93, 402-406.

16. Ellegren, H. Microsatellites: Simple sequences with complex evolution. Nat. Rev. Genet. 2004, 5, 435-445.

17. Hakki, E.; Christo, P.; Luchezar, K.; Mahinur, A. Isolation of wheat microsatellite DNA fragments by hybridization selection. Bulg. J. Plant Physol. 2002, 28, 3-10.

18. Roder, M.S.; Plaschke, J.; Konig, S.U.; Borner, A.; Sorreils, M.E. Abundance, variability and chromosomal location of microsatellites in wheat. Mol. Gen. Genet. 1995, 246, 327-333.

19. Kantety, R.V.; La Rota, M.; Matthews, D.E.; Sorrells, M.E. Data mining for simple sequence repeats in expressed sequence tags from barley, maize, rice, sorghum and wheat. Plant Mol. Biol. 2002, 48, 501-510.

20. Lunt, D.H.; Hutchinson, W.F.; Carvalho, G.R. An efficient method for PCR-based isolation of microsatellite arrays (PIMA). Mol. Ecol. 1999, 8, 891-894.

21. NCBI. Available online: http://www.ncbi.nlm.nih.gov/genbank/ (accessed on 14 October 2011).

22. Rozen, S.; Skaletsky, H.J. Primer3 on the WWW for General Users and for Biologist Programmers. In Bioinformatics Methods and Protocols: Methods in Molecular Biology; Krawetz, S., Misener, S., Eds.; Humana Press: Totowa, NJ, USA, 2000; pp. 365-386.

23. Kalinowski, S.T.; Taper, M.L.; Marshall, T.C. Revising How the computer program CERVUS accommodates genotyping error increases success in paternity assignment. Mol. Ecol. 2007, 16, 1099-1106.

24. Rousset, F. Genepop'007: A complete reimplementation of the Genepop software for Windows and Linux. Mol. Ecol. Resour. 2008, 8, 103-106.

(C) 2011 by the autHors; licensee MDPI, Basel, Switzerland. This article is an open access article distributed under the terms and conditions of the Creative Commons Attribution license (http://creativecommons.org/licenses/by/3.0/). 